\title{
Traduire
}

Une eutre perspective sur $r$ tatadciction

Revue française de la traduction

$244 \mid 2021$

Des jeux et des mots

\section{Les multiples facettes de la traduction de jeux}

\section{Sandrine Guyennet}

\section{(2) OpenEdition}

\section{Journals}

Édition électronique

URL : https://journals.openedition.org/traduire/2274

ISSN : 2272-9992

Éditeur

Société française des traducteurs

Édition imprimée

Date de publication : 15 juin 2021

Pagination : $9-21$

ISSN : 0395-773X

Référence électronique

Sandrine Guyennet, «Les multiples facettes de la traduction de jeux », Traduire [En ligne], 244 | 2021, mis en ligne le 15 juin 2021, consulté le 01 juillet 2021. URL : http://journals.openedition.org/traduire/ 2274 


\section{Les multiples facettes de la traduction de jeux}

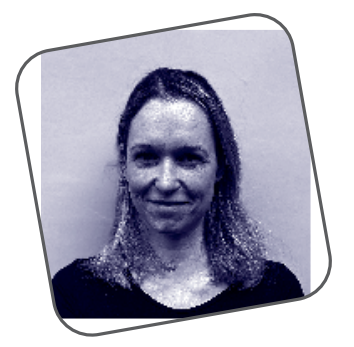

\section{Sandrine Guyennet}

Il arrive souvent qu'on me dise en apprenant ma profession: "J'adore les jeux vidéo et je parle anglais! Tu peux me trouver des jeux à traduire? »Peut-être plus que d'autres spécialisations, la mienne attire les amateurs qui pensent, à tort, qu'il suffit d'aimer les jeux vidéo pour les traduire correctement. Ce problème est attisé par certains studios de développement qui comptent sur leur communauté de fans pour traduire leurs jeux gratuitement, ce qui peut être un désastre. Connaître le vocabulaire des gamers et des jeux n'est qu'un aspect mineur du métier, et peut-être le plus facile à apprendre. En plus de maîtriser parfaitement la langue cible et la culture des pays concernés, ce qui est la base du bagage de tous les traducteurs et de toutes les traductrices, il faut aussi se soumettre à toutes les contraintes techniques spécifiques à la localisation de logiciels, connaître les réglementations locales en matière de publication d'œuvres vidéoludiques et maîtriser les œuvres cultes de la culture geek. 


\section{Traducteur littéraire}

La traduction de jeux vidéo est une spécialisation qui se rapproche sur certains points de la traduction littéraire. En effet, pour permettre l'immersion des joueuses et joueurs dans l'univers particulier d'un jeu, on ajoute à celvi-ci du flavor text, c'est-à-dire du texte qui n'est pas essentiel pour l'action, mais qui apporte des éléments narratifs. Il peut s'agir de pages d'un journal intime trouvé dans les bâtiments désaffectés d'un jeu de tir et racontant le début de l'apocalypse zombie, ou bien d'une encyclopédie des êtres magiques détaillant les soixante races fantastiques que le joveur peut incarner dans un RPG' japonais, ou encore d'un concours d'insultes aux jeux de mots affligeants dans un jeu d'aventure loufoque. Le flavor text est essentiel pour tracer les contours du jeu et lui conférer une atmosphère. II s'agit de donner un passé aux personnages et une histoire au monde dans lequel ils évolvent. Si c'est réussi, on a "l'impression d'y être». Les MMORPG (jeux de rôle massivement multijoueur), dont l'action se déroule souvent à une époque médiévale fictive, peuvent imposer de traduire des centaines de pages de chroniques historiques, de légendes et de mythologie.

Certains jeux sont directement inspirés d'œuvres littéraires et bourrés de citations. J'ai notamment travaillé sur plusieurs jeux d'objets cachés tirés de nouvelles d'Edgar Allan Poe, sur un jeu d'exploration en réalité virtuelle inspiré d'Alice au Pays des Merveilles de Lewis Carroll et sur un jeu de réflexion reprenant La Métamorphose de Franz Kafka. Dans le cas de références classiques ou célèbres, il convient de mettre la main sur les traductions existantes afin de retrouver notamment les citations et les noms propres, mais aussi de reproduire le style et le ton. Pour les inspirations plus récentes ou obscures, on peut être amené à retraduire, souvent pour des questions de droits d'auteur, ou parce que les traductions sont introuvables ou inexistantes.

1. Les RPG (Role-Playing Games) résultent du portage en jeu vidéo des jeux de rôle traditionnels se jouant sur table. Dans les RPG, le personnage jouable, souvent personnalisable, part à l'aventure, évolue et gagne des compétences au fil du jeu, effectue des quêtes et recrute des compagnons. Parmi les franchises emblématiques du genre, on peut citer Final Fantasy et Dragon Quest. 


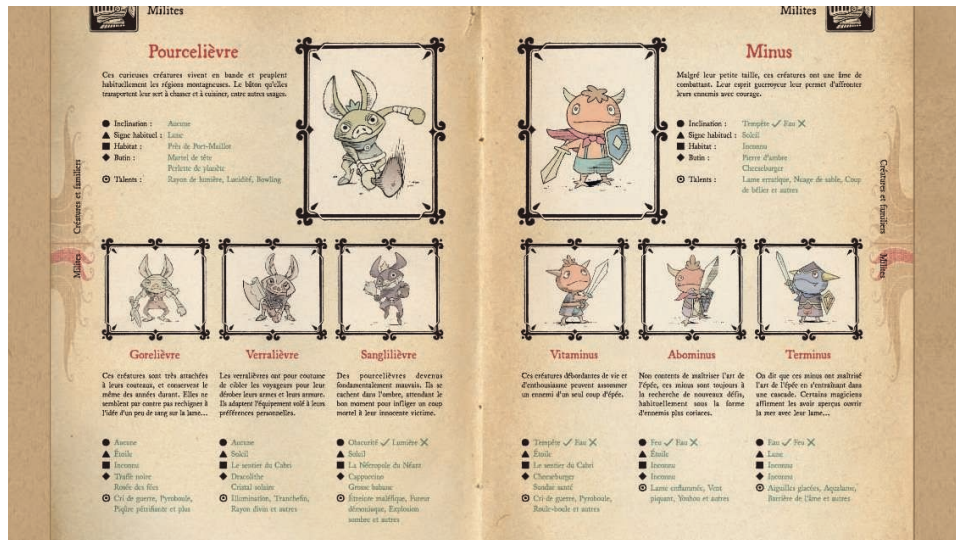

Dans Ni no Kuni: La Vengeance de la Sorcière Céleste, un almanach de 340 pages, intégrale-

\section{As du marketing}

Avant tout, le rôle essentiel de la traductrice ou du traducteur est de favoriser les ventes du jeu sur le marché local. L'accent est donc mis sur la qualité des textes marketing. Pour les grosses productions, il s'agit du site web dédié, d'éventuels articles de presse, de la jaquette de la boîte, de publicités destinées aux magazines, à la télévision ou aux réseaux sociaux, des publications du ou de la community manager, ainsi que du texte d'accroche pour les sites de vente en ligne. Il arrive souvent que les petits développeurs de jeux, qui disposent d'un budget restreint, décident de faire appel à un traducteur professionnel uniquement pour les pages des sites de vente, comme l'App Store d'Apple, le Play Store de Google ou Steam. La description du jeu est traduite, mais pas le jeu lui-même. Peu importe aux studios que les joueurs aient effectivement jové au jeu: ils recherchent simplement le plus grand nombre de téléchargements possible. Chaque téléchargement leur permet d'obtenir un paiement, que ce soit sous la forme d'un achat classique, ou bien, pour les jeux mobiles "gratuits», par la collecte des données à caractère personnel des utilisateurs ou la diffusion de publicités intégrées au jeu. Le traducteur a pour mission d'augmenter les ventes en boostant la visibilité du jeu, notamment en utilisant 
des techniques de référencement $\left(\mathrm{SEO}^{2}\right)$, mais il faut aussi «faire envie», quitte à parfois survendre le jeu en promettant des fonctionnalités qui n'en font pas partie.

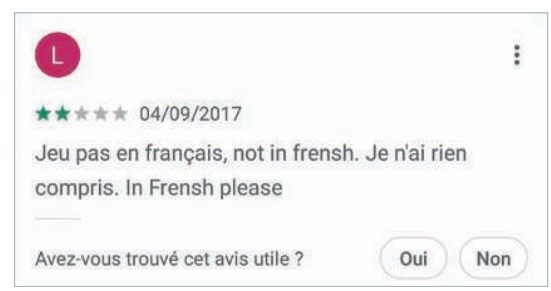

Cette joueuse a cru que le jeu était traduit en français, après en avoir lu la description en français dans le Play Store de Google.

\section{Traducteur audiovisuel}

À bien des égards, l'activité des traductrices et traducteurs de jeux vidéo est similaire à celle de leurs confrères et consœurs qui se spécialisent dans le domaine audiovisuel. Dans certaines grosses productions, les personnages ont la voix de véritables acteurs et actrices. Dans ce cas, comme pour les films, il faut fournir un script traduit accompagné d'indications de ton et de prononciation. Une erreur de traduction dans un script envoyé à l'enregistrement peut parfois être désastreuse. Quand cela n'a pas d'impact sur l'action du jeu, les développeurs, en raison des contraintes de temps et de budget, ont tendance à laisser les erreurs, en misant sur le fait qu'elles passeront inapercues. En effet, tant que l'on n'a pas le texte source sous les yeux, il est difficile de repérer une erreur de traduction qui ne porte pas sur un élément visible à l'écran, qui n'a pas trait à une action ou qui est prononcée par un personnage secondaire obscur que le joueur a peu de chances de rencontrer au cours de son aventure.

À l'inverse, une erreur de traduction portant sur une action du jeu, invitant par exemple à se rendre à la gare au lieu du commissariat (tous deux station en anglais), bloquerait complètement le joveur. II faudrait alors allover du temps et de

2. Le SEO (Search Engine Optimization) est l'art de positionner un site, une page web ou une application dans les premiers résultats naturels des moteurs de recherche. Quand un utilisateur effectue une recherche, les sites qui sont les mieux référencés apparaissent en premier dans la liste des résultats. 
l'argent à sa correction. Dans ce cas, on blâme souvent les traducteurs et traductrices. Or il est parfois impossible, par manque de temps, de tout relire avec la même attention. Cela est d'autant plus vrai pendant le crunch ${ }^{3}$, la période de travail intense et épuisante qui précède la sortie ou la présentation d'un jeu, et pendant laquelle il faut rattraper tout le retard pour tenir les délais. En matière de relecture, on se concentre donc en priorité sur ce qui est difficile et coûteux à modifier ultérieurement: le script pour le doublage, et le texte graphique, c'est-à-dire dessiné. On peut faire preuve d'une plus grande souplesse avec le texte facilement modifiable pendant la phase de test, ainsi que pour le flavor text, qui n'a pas d'impact direct sur l'action.

Dans les jeux vidéo, les voix ne sont parfois pas doublées par souci d'économie, mais quasiment toujours sous-titrées pour des raisons d'accessibilité. De ce point de vue, les contraintes sont donc identiques à celles de la traduction audiovisuelle: synchronisation des paroles avec les mouvements des lèvres pour le doublage, nombre de caractères et nombre de lignes maximum pour le sous-titrage. En revanche, les sous-titres posent une difficulté supplémentaire dans les jeux, parce qu'ils apparaissent dans le feu de l'action et doivent donc être courts et compréhensibles du premier coup d'œil. Si le joveur comprend ce qu'il doit faire un quart de seconde trop tard, il risque de ne pas agir à temps.

\section{Spécialiste de la localisation}

Un jeu est avant tout un programme informatique, c'est-àdire un ensemble de lignes de code informatique. Le texte du jeu lui-même, que le joueur verra à l'écran, se niche généralement au beau milieu des bribes de code, des balises et des variables. En traduisant, il ne faut surtout pas casser la fonctionnalité du jeu. Modifier le code par inadvertance, par exemple en remplaçant toutes les apostrophes droites par des apostrophes courbes lors d'un malheureux chercher/ remplacer, peut rendre le jeu complètement inutilisable. 
Le code ne s'exécute plus, et le jeu peut ne pas se lancer. Dans les cas les moins graves, des parties de balises risquent d'apparaître à l'écran.

Les traductrices et traducteurs de jeux doivent donc savoir travailler "autour» du code sans le casser. Les meilleurs connaissent la signification des balises les plus connues (comme 〈br〉 ou \n qui signifient un retour à la ligne), et comprennent comment fonctionne le code, c'est-à-dire dans quel ordre les phrases vont apparaitre et quelles actions du jeu permettent de déclencher leur affichage. Cela permet aussi de déplacer les variables dans la traduction en toute sécurité. Il est aussi particulièrement utile de savoir paramétrer les outils TAO pour qu'ils reconnaissent les balises et variables peu communes ou créées par le développeur.

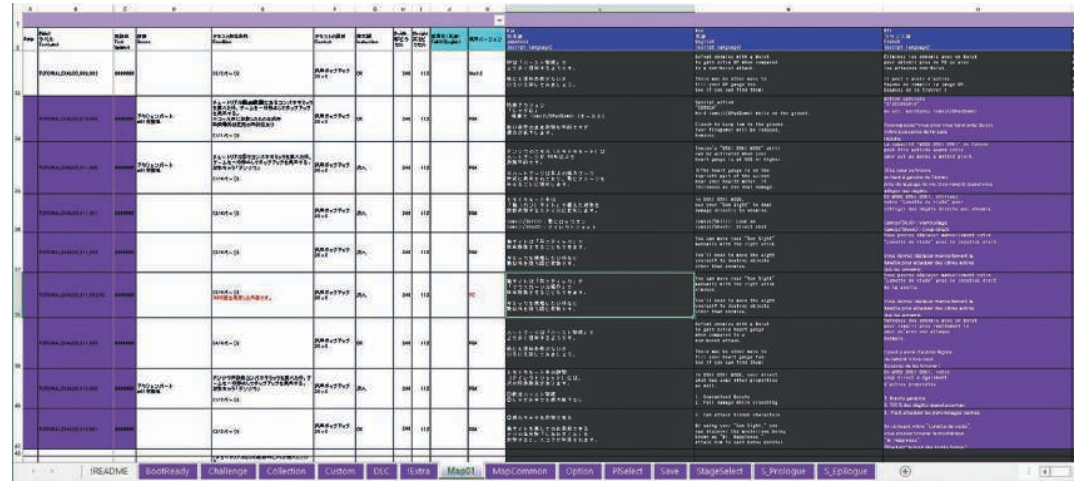

Aperçu d'un fichier Excel aux multiples onglets pour la traduction d'un jeu en dix langues.

Une autre contrainte propre aux jeux est l'espace alloué au texte. On a vu que les sous-titres sont, comme à la télévision, limités en nombre de caractères, mais ce n'est pas le seul paramètre à prendre en compte. Tous les textes de l'interface du jeu (menus, messages système, boutons) figurent sur des éléments graphiques de taille limitée, en particulier sur les petits écrans des consoles portables et les téléphones.

La traduction des messages système constitue un autre aspect technique. Chaque plateforme a sa terminologie, approuvée par le fabricant. Par exemple, Nintendo a choisi le mot «Bouton» pour ses consoles et Sony le mot «Touche». La traduction française du message "Press any key to continue» différera donc pour la PlayStation ${ }^{\oplus 5}$ et la Nintendo Switch ${ }^{\mathrm{TM}}$. 
Les symboles de marque déposée ont aussi leur importance. Tous les jeux passent un test de conformité des termes et messages officiels avant d'être mis en vente sur une plateforme, et la moindre erreur entraînera un refus.

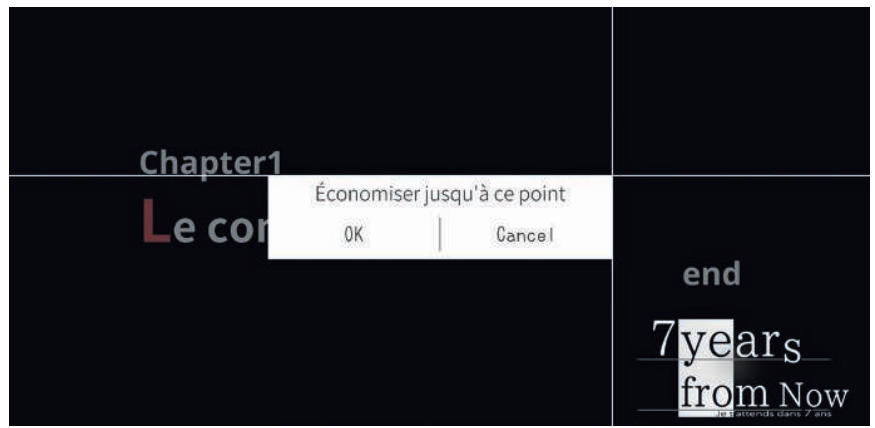

Ce genre de traduction erronée (au lieu de "Sauvegarder la progression») peut conduire au refus de publication du jeu. La majorité des messages système sont préapprouvés pour toutes les langues par les gros éditeurs, notamment pour des raisons légales, et doivent être utilisés tels quels, à la virgule près.

Un autre aspect propre à la localisation, controversé mais bien plus créatif, est l'adaptation ou non des thèmes et éléments abordés. Doit-on remplacer une métaphore de baseball, très américaine, par une image propre au football? Si un personnage se remémore avec nostalgie les petits plats que lui préparait sa grand-mère, doit-on les européaniser quand il s'agit de plats japonais peu connus en Occident? Le débat fait rage. Si on adapte trop, les joveurs, en particulier ceux qui sont familiers du pays en question, se plaignent de surlocalisation. Une adaptation insuffisante empêche l'immersion - la suspension de l'incrédulité (suspension of disbelief) - des joveurs qui ne connaissent pas le pays et ne comprennent pas de quoi on parle. La suspension volontaire de l'incrédulité est le concept selon lequel on passe outre les aberrations du monde du jeu pour s'autoriser une immersion totale. Par exemple: on peut transporter n'importe quoi dans un inventaire illimité en poids et en volume, que ce soient des litres de liquides sans récipient, une échelle, un cheval, ou encore plusieurs dizaines d'armes lourdes; on peut parcourir des centaines de kilomètres à pied, sans manger ni boire, et le fait de marcher nous redonne des forces; on visite des mondes imaginaires peuplés de créatures qui n'existent pas et on voyage dans l'espace, dans le temps ou entre les mondes. 
Malgré tout cela, on oublie le monde réel, "on s'y croirait». La suspension de l'incrédulité ne fonctionne que dans un monde cohérent. Il faut à tout prix éviter qu'elle soit brisée par quelque chose qui arrive comme un cheveu sur la soupe, comme un mot qui est resté en anglais ou une erreur de traduction, par exemple une pipe qui s'appelle «tuyau» (tous deux pipe en anglais). Le concept s'applique aussi aux autres œuvres de fiction, comme les films ou les romans.

Si un personnage de jeu mentionne un dessin animé qui a bercé son enfance, et que ce dessin animé a été traduit, mais est passé inapercu en France, on peut préférer remplacer l'alIusion par quelque chose qui a été plus populaire dans l'Hexagone. On touche ici à un autre problème: où sera utilisée la traduction française? En France uniquement? Ou également en Belgique, au Canada ou au Cameroun? Les références culturelles ne seront pas les mêmes d'un pays à l'autre.

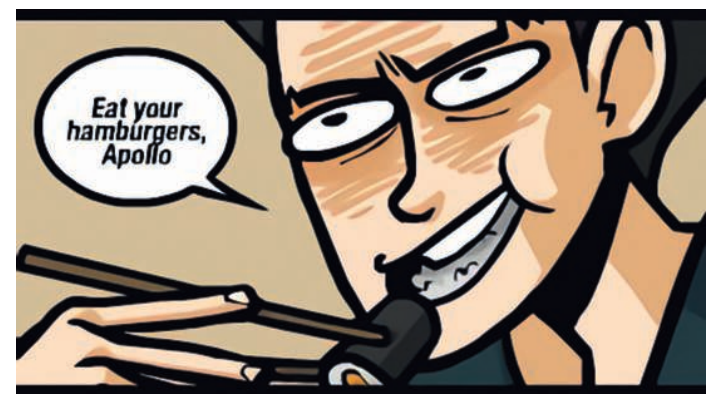

Un exemple de localisation controversée est le remplacement de "ramens" par "hamburgers" dans le jeu Phoenix Wright: Ace Attorney, dans le cadre d'une localisation transposant l'action d'une ville japonaise à Los Angeles. Le mème ci-dessus fait référence à ce choix de localisation ${ }^{4}$.

\section{Spécialiste de la culture populaire}

Un élément essentiel du bagage des traducteurs et traductrices de jeux vidéo est leur excellente connaissance de la culture populaire. Traditionnellement, les jeux sont truffés de références plus ou moins subtiles et plus ou moins obscures à un univers geek. Certains thèmes reviennent si souvent qu'on

4. HSU Janet, Localization and Ace Attorney, blog de Capcom (anglais) : https://www.capcom-unity.com/ace_attorney/blog/2014/11/21/localization-and-ace-attorney, consulté le $28 / 04 / 2021$. 
frise le cliché: la mythologie nordique, les univers Star Wars et Star Trek, les œuvres de Lovecraft, de Pratchett ou des Monty Pythons, ou encore Retourvers le futur ou Harry Potter.

\begin{tabular}{|l|l|}
\hline $\begin{array}{l}\text { Reference to Marie Kondo's } \\
\text { quote. }\end{array}$ & $\begin{array}{l}\text { When over-encumbered, hold your extra gear and think ... Indoes } \\
\text { this bring me joy? }\end{array}$ \\
\hline $\begin{array}{l}\text { Reference to Monkey Island. } \\
\text { Talking to the player. Can be } \\
\text { said by a male or female. }\end{array}$ & You fight like a cow! \\
\hline
\end{tabular}

Dans l'idéal, le studio prépare le texte source qu'il confie en traduction en ajoutant des notes explicatives afin qu'aucune référence ne passe inaperçue.

Quand le studio ne laisse pas de notes explicatives, il faut savoir repérer les références populaires. Un titre de niveau paraît étrange? II cache peut-être un jeu de mots basé sur une citation tirée d'un film ou sur le dernier mème ${ }^{5}$ populaire sur internet. Dans ce cas, et si on ne peut pas contacter le développeur pour demander plus de précisions, mieux vaut jouer la carte de la sécurité et multiplier les recherches. Sinon, on risque de générer une nouvelle traduction de citations que les futurs joueurs et joueuses connaissent sur le bout des doigts. Cela peut être un atout de taille de "passer sa vie sur internet», d'être constamment à l'affût sur Reddit, Twitch, Discord ou Twitter, pour ne rien rater des derniers buzz et mèmes qui ont pu influencer le développement du jeu. C'est aussi le moyen de rester au contact des gamers et de leur vocabulaire. S'ils ont pris l'habitude d'utiliser un terme anglais pour désigner telle ou telle option, autant les suivre.

Une fois qu'on a repéré les références aux mèmes, aux émissions de télévision, aux films, aux événements sportifs ou culturels, comment gérer leur traduction? Si l'œuvre a également paru en français, on essaiera d'en trouver la traduction officielle. Le but est de produire chez celles et ceux qui jouent en français les impressions ressenties par celles et ceux qui jouent dans la langue d'origine. Pour cela, il peut parfois être nécessaire d'adapter, plutôt que de traduire, les références culturelles, métaphores et jeux de mots.

5. «Concept (texte, image, vidéo) massivement repris, décliné et détourné sur internet de manière souvent parodique, qui se répand très vite, créant ainsi le buzz.» (Larousse, Mème, https://www.larousse.fr/dictionnaires/francais/mème, consulté le 10/02/2021.) 
À ces connaissances très spécifiques doit s'ajouter une culture générale solide pour éviter de perdre trop de temps en recherches, de faire également perdre du temps au studio de développement avec des questions qui peuvent être évitées, ou même de vexer une partie des joueurs en commettant des erreurs de traduction. Une bonne culture générale peut permettre de déchiffrer le véritable sens d'un texte rédigé par des personnes dont l'anglais n'est pas la langue maternelle.

\section{Purchase items above? «phid="1">They will be used immodestly after purchase.}

L'anglais signifie ici «Acheter les objets ci-dessus? Ils seront utilisés avec indécence après l'achat». En réalité, "immodestly» est une erreur pour «immediately», et le véritable sens de la phrase est «lls seront utilisés immédiatement après l'achat».

\section{Chercheur avisé}

Comme on vient de le voir, les traductrices et traducteurs de jeu doivent avoir ce qu'on appelle parfois en anglais un bon "google-fu», c'est-à-dire maîtriser les moteurs de recherche autant que les grands maîtres du kung-fu maîtrisent leur art. La particularité des recherches pour des jeux vidéo réside sans doute dans la multitude de domaines abordés. On peut être amené à se renseigner sur les armes automatiques, la propulsion de fusées, le golf, les grades de l'armée américaine, les recettes de cuisine coréennes, les règles du hockey sur glace, le code de la route australien, les techniques de sculpture sur bois, les différentes espèces de colibri, le vocabulaire des DJ, les plantes médicinales ou encore les formations défensives des légions romaines. Tous ces exemples sont authentiques! Entre collègues, on évoque souvent I'hypothétique agent du FBI qui viendrait toquer à la porte à cause de nos recherches douteuses. Cependant, la multitude de thèmes abordés dans les jeux permet d'apprendre sans cesse sur des sujets incroyablement variés, ce qui est très enrichissant.

Les recherches restent bien moins poussées que celles que ferait un traducteur spécialisé dans les domaines euxmêmes, autant du point de vue de la complexité (un joueur «lambda» doit être en mesure de comprendre la majeure 
partie du jeu) que du nombre de termes à vérifier. L'expertise médicale requise pour traduire Trauma Center, un jeu de simulation d'opération chirurgicale pour Nintendo DS, est infiniment moindre que celle nécessaire pour traduire des documents destinés à de véritables chirurgiens. Une erreur de traduction aura aussi un impact beaucoup moins dramatique, bien évidemment.

Dans le cas d'un sujet très pointu, les recherches ne suffisent pas, ou prendraient beaucoup trop de temps, et mieux vaut passer la main à un spécialiste. Pour cette raison, je refuse de travailler sur la plupart des jeux de sport réalistes et des jeux de simulation de pilotage et de conduite. Néanmoins, pour un jeu de simulation de golf très pointu, j'ai travaillé à quatre mains avec un journaliste sportif spécialisé dans ce sport.

\begin{tabular}{|c|c|}
\hline $\begin{array}{l}\text { The } 207-y a r d 17 \text { th plays upt } \\
\text { shaped putting surface that } \\
\text { the teeing ground and is sur } \\
\text { bunkers. The putting green } \\
\text { relatively shallow in depth a } \\
\text { ridge that separates two dis } \\
\text { sure to be an exciting hole t }\end{array}$ & $\begin{array}{l}\text { ouré de } \\
\text { ent visible } \\
\text { fond et propose } \\
\text { eux par une } \\
\text { 'un des trous } \\
\text { un rôle décisif } \\
\text { tournoi. }\end{array}$ \\
\hline $\begin{array}{l}\text { The home hole provides a dramatic finish. The } \\
\text { downhill tee shot on this } 411 \text {-yard par } 4 \text { gives } t \\
\text { player a choice - lay up short of the deep cluste } \\
\text { bunkers or be more aggressive off the tee and } \\
\text { attempt to drive his ball between or beyond th }\end{array}$ & $\begin{array}{l}\text { Au départ } \\
\text { un choix } \\
\text { x groupes } \\
\text { bien } \\
\text { iver. } \\
\text { incliné de }\end{array}$ \\
\hline
\end{tabular}

\section{Spécialiste de la réglementation locale}

Chaque pays a ses propres réglementations en matière de publication de jeux vidéo. En Europe, le classement PEGl ${ }^{6}$ détermine l'âge minimum pour jover à un jeu, en fonction de son contenu: langage vulgaire, nudité, violence, mais aussi consommation d'alcool, de tabac ou de stupéfiants, scènes trop effrayantes ou achats intégrés.

Dans certains pays, il faut aussi anticiper la censure en évitant les thèmes tabous. Par exemple, il ne sera pas possible de publier en Chine un jeu présentant le Tibet comme un pays indépendant. L'Arabie saoudite interdit les symboles

6. Pan European Game Information (https://pegi.info/fr). 
comme la croix ou l'étoile à six branches. Au Venezuela, tous les jeux violents sont prohibés. En France, aucun jeu n'a jamais été interdit, et il est donc rarement nécessaire d'apporter de grosses modifications. Un léger ajustement du texte, par exemple la suppression de certaines grossièretés, peut suffire à faire passer un jeu de la catégorie «Moins de 16 ans» à "Moins de 12 ans», ce qui élargit le public cible et augmente potentiellement les ventes. C'est au studio de développement, éclairé par les conseils de l'équipe de traduction, qu'en revient la décision.

Dans le délicat domaine des réglementations et coutumes locales, le dialogue avec le client s'avère une fois encore essentiel. Le traducteur a un rôle de consultant culturel: il doit identifier les parties problématiques du texte, les communiquer au client, évaluer l'impact potentiel sur les ventes et l'image de marque, et proposer des solutions.

À ce propos, certaines problématiques qui ne sont ni interdites par la loi ni prises en compte dans les classements doivent toutefois être identifiées et communiquées au client. Par exemple, tout ce qui relève de la misogynie, de la grossophobie ${ }^{7}$ du dénigrement d'un segment de la population, peut entraîner de mauvaises évaluations du jeu, voire un bad

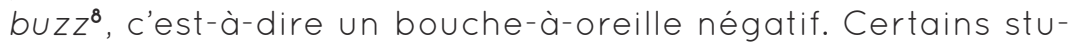
dios dont la réputation a été entachée ont perdu la confiance des joueurs et ne s'en sont jamais relevés.

\section{2) $\star * * * * 13$ octobre 2020

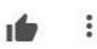 \\ Grossophobe. Ce jeu est une insulte à la diversité des corps. Ça na pas sa place, surtout dans une app éducative. C'est une honte.}

Malgré mon insistance, le studio de développement a choisi de ne pas supprimer du jeu un niveau à la grossophobie flagrante.

7. Comportement discriminatoire et stigmatisant envers les personnes en surpoids.

8. Définitions marketing, Bad buzz, https://www.definitions-marketing.com/definition/badbuzz/, consulté le 28/04/2021. 


\section{Conclusion}

On l'a vu, le travail des traducteurs et traductrices de jeux vidéo nécessite une grande polyvalence. Technique et créativité occupent une même place dans leurs traductions et c'est peut-être pour cela que cette spécialisation attire des linguistes aux profils très variés et issus de formations diverses. Les diplômes de localisation de jeux vidéo ne sont apparus que tout récemment dans les cursus des universités et des écoles spécialisées. Dans quelques années, nous aurons le recul nécessaire pour étudier l'impact de ces formations dédiées sur les traductions professionnelles de jeux - une amélioration de la qualité des traductions ou, à l'inverse, une trop forte harmonisation des styles et des termes?

sandrine@guyennet.fr

Sandrine Guyennet exerce en tant que traductrice de jeux vidéo de l'anglais au français depuis 2008 . Diplômée du master de traduction de l'université de Westminster à Londres, elle a débuté en interne dans les services de localisation d'éditeurs de jeux, en Angleterre, puis en Irlande, avant de s'installer à son compte. Après dix ans d'expatriation, elle est de retour en France et vit à Bordeaux. Sandrine s'investit dans la sphère de la localisation de jeux vidéo et rédige des articles sur ce thème pour son blog (https:// thefrenchalizer.com/blog). Elle est membre de la SFT depuis 2016 et fait partie des mentors du programme Boussole depuis 2020. 